\title{
A mechanical model of tunnelling
}

\author{
Amaury Mouchet \\ Laboratoire de Mathématiques et Physique Théorique, \\ Université François Rabelais de Tours - CNRS (UMR 6083), \\ Fédération Denis Poisson, \\ Parc de Grandmont 37200 Tours, France. \\ mouchet@phys.univ-tours.fr
}

June 4, 2022

\begin{abstract}
It is shown how the model which was introduced in 1 allows to mimic the quantum tunnelling between two symmetric one-dimensional wells.

Following the publication of [1, some readers asked me to provide more details on the concluding remark about tunnelling (first paragraph of $\S 6$ ). Strictly speaking, tunnelling is a quantum phenomenon that refers to any process that is classically forbidden (i.e. cannot be understood from the real solutions of the Hamilton equations). The paradigmatic example (that can be found in many textbooks, like 21) is given by the oscillations of a quantum system between two one-dimensional potential wells separated by a barrier whose maximal energy is larger than the energy of the system. As understood as early as 1927 by Hund [3, it relies on the existence of some evanescent Schrödinger waves connecting the two wells. Therefore, it is perfectly justified to consider a purely classical mechanical model to illustrate tunnelling provided it exhibits the appropriate evanescent waves (we do not intend here to compare the classical model with its quantum analogue). In optics, where evanescent waves can be easily created with dielectric materials, the analogue of quantum tunnelling has already been considered, for instance in [4].

To mimic the double-well symmetric situation, let us consider two identical oscillators coupled to the Klein-Gordon string (as described in [1]) and located at $x= \pm a / 2(a>0)$. When the oscillation frequency of the free oscillators $\Omega_{0}$ is below the "infra-red" cut-off on the Klein-Gordon string $\omega_{0}$, there are two stable modes, one symmetric and one antisymmetric whose corresponding frequencies are denoted by $\omega_{+}$and $\omega_{-}$respectively : If the two oscillators were infinitely far away, we would get $\omega_{+}=\omega_{-}=\omega_{b}$ given by (42) of [1] but at finite distance the string connecting the two oscillators lifts the degeneracies between the two frequencies : the splitting $\Delta \omega \stackrel{\text { def }}{=} \omega_{-}-\omega_{+} \neq 0$ represents the frequency of the beating between the two oscillators. At these frequencies $\omega_{ \pm}$, both below $\omega_{0}$, no energy transport along the string is allowed on the average (equation (9) of 1]) and however some energy can be exchanged between the two oscillators.

This is similar with the quantum situation: for the quantum symmetric double-well potential, the true eigenstates are delocalised between the two wells
\end{abstract}


with energies $E_{+}$and $E_{-}$; a state that is localised in one well only is a linear superposition of a symmetric and an antisymmetric state and oscillate back and forth between the two wells at a frequency $\left(E_{-}-E_{+}\right) / \hbar$.

The tunnelling splitting is expected to be exponentially small with the distance between the two oscillators [5] as can be proved by the following computation:

For $x<-a / 2$, we keep an evanescent wave on the string with the form $D_{-} \mathrm{e}^{\mathrm{i}(-k(x+a / 2)-\omega t)}$ with $k=\mathrm{i} \sqrt{\omega_{0}^{2}-\omega^{2}}$ whereas for $-a / 2<x<0$ we have $C_{+} \mathrm{e}^{\mathrm{i}(k(x+a / 2)-\omega t)}+D_{+} \mathrm{e}^{\mathrm{i}(-k(x+a / 2)-\omega t)}$. The complex coefficients $C_{+}$and $D_{+}$ are linearly related to $D_{-}$with the matrix $T(\omega)$ given by (23-26) of [1]. The antisymmetric mode corresponds to an odd wavefunction that vanishes at $x=0$ : $C_{+} \mathrm{e}^{\mathrm{i} k a / 2}+D_{+} \mathrm{e}^{-\mathrm{i} k a / 2}=0$. The symmetric mode corresponds to an even wavefunction whose spatial derivative vanishes at $x=0: C_{+} \mathrm{e}^{\mathrm{i} k a / 2}-D_{+} \mathrm{e}^{-\mathrm{i} k a / 2}=0$. In both cases, a non-trivial solution can be found if $\rho= \pm \mathrm{e}^{-\mathrm{i} k a}$. The frequencies $\omega_{ \pm}$must be the solutions of the two equations respectively

$$
1+\frac{2 \sqrt{\omega_{0}^{2}-\omega^{2}}}{\kappa} \frac{\omega^{2}-\Omega_{\kappa}^{2}}{\omega^{2}-\Omega_{0}^{2}}=\mp \mathrm{e}^{-a \sqrt{\omega_{0}^{2}-\omega^{2}}} .
$$

If the right hand side vanished ( $a \rightarrow+\infty$ while keeping fixed all the other parameters), we would recover the equation that furnishes $\omega_{b}$ (see equation (38) of [1). For finite $a$, in the case where the right hand side is small $\left(a \sqrt{\omega_{0}^{2}-\Omega_{0}^{2}} \gg\right.$ 1 ), the first order expansion in the coupling parameter $\kappa$ provides

$$
\Delta \omega \simeq \frac{\kappa^{2}}{2 \Omega_{0} \sqrt{\omega_{0}^{2}-\Omega_{0}^{2}}} \mathrm{e}^{-a \sqrt{\omega_{0}^{2}-\Omega_{0}^{2}}} .
$$

\section{References}

[1] A. Mouchet, Eur. J. Phys. 29, 1033 (2008).

[2] A. Messiah, Mécanique Quantique (2 vol.) (Dunod, Paris, 1964), english translation: North-Holland (Amsterdam).

[3] E. Merzbacher, Phys. Today 55, 44 (2002).

[4] J. U. Nöckel and A. D. Stone, Nature 385, 45 (1997).

[5] A. Garg, Amer. J. Phys. 68, 430 (2000). 